\title{
Versatile applications of transcriptional pulsing to study mRNA turnover in mammalian cells
}

\author{
CHYI-YING A. CHEN, ${ }^{1}$ YUKIKO YAMASHITA, ${ }^{1,2}$ TSUNG-CHENG CHANG, ${ }^{3}$ AKIO YAMASHITA, ${ }^{2}$ \\ WENMIAO ZHU, ZHENPING ZHONG, and ANN-BIN SHYU \\ Department of Biochemistry and Molecular Biology, The University of Texas Medical School, Houston, Texas 77030, USA
}

\begin{abstract}
Development of transcriptional pulsing approaches using the c-fos and Tet-off promoter systems greatly facilitated studies of mRNA turnover in mammalian cells. However, optimal protocols for these approaches vary for different cell types and/or physiological conditions, limiting their widespread application. In this study, we have further optimized transcriptional pulsing systems for different cell lines and developed new protocols to facilitate investigation of various aspects of mRNA turnover. We apply the Tet-off transcriptional pulsing strategy to investigate ARE-mediated mRNA decay in human erythroleukemic K562 cells arrested at various phases of the cell cycle by pharmacological inhibitors. This application facilitates studies of the role of mRNA stability in control of cell-cycle dependent gene expression. To advance the investigation of factors involved in mRNA turnover and its regulation, we have also incorporated recently developed transfection and siRNA reagents into the transcriptional pulsing approach. Using these protocols, siRNA and DNA plasmids can be effectively cotransfected into mouse NIH3T3 cells to obtain high knockdown efficiency. Moreover, we have established a tTA-harboring stable line using human bronchial epithelial BEAS-2B cells and applied the transcriptional pulsing approach to monitor mRNA deadenylation and decay kinetics in this cell system. This broadens the application of the transcriptional pulsing system to investigate the regulation of mRNA turnover related to allergic inflammation. Critical factors that need to be considered when employing these approaches are characterized and discussed.
\end{abstract}

Keywords: mRNA turnover; deadenylation; transcriptional pulsing; siRNA knockdown; cell cycle

\section{INTRODUCTION}

The recognition of mRNA turnover as a fundamental mechanism for governing eukaryotic gene expression (Wilusz et al. 2001; Meyer et al. 2004; Parker and Song 2004) has mandated the development of reliable, rigorous, and "user-friendly" methods to accurately measure changes in mRNA stability in mammalian cells. Messenger RNA stability is often studied indirectly by monitoring changes in the steady-state level of mRNA in the cytoplasm; in this case, changes in mRNA abundance are assumed to reflect only mRNA degradation, an assumption that is not always correct (for review, see Ross 1995; Loflin et al. 1999b). Direct measurements of mRNA decay rate can be per-

\footnotetext{
${ }^{1}$ These authors contributed equally to this work.

Present addresses: ${ }^{2}$ Yokohama City University School of Medicine, 3-9 Fuku-ura, Kanazawa, Yokohama, 236-0004, Japan; ${ }^{3}$ The McKusickNathans Institute of Genetic Medicine, Johns Hopkins University School of Medicine, Baltimore, MD 21205, USA.

Reprint requests to: Ann-Bin Shyu, Department of Biochemistry and Molecular Biology, The University of Texas Medical School, Houston, TX 77030, USA; e-mail: Ann-Bin.Shyu@uth.tmc.edu; fax: (713) 500-0652.

Article published online ahead of print. Article and publication date are at http://www.rnajournal.org/cgi/doi/10.1261/rna.663507.
}

formed in a number of ways, including kinetic labeling techniques and the use of transcriptional inhibitors, although these techniques are tedious and often introduce significant changes in cell physiology (for review, see Harrold et al. 1991; Belasco and Greenberg 1993; Ross 1995). For example, actinomycin D, which interferes with transcription by intercalating into DNA, and 5,6-dichloro$1 \beta$-D-ribofuranosylbenzimidazole (DRB), which interacts with the RNA polymerase II transcription apparatus, have been widely used to block transcription to analyze mRNA decay kinetics. However, both agents have a profound impact on cellular physiology and perturb the stability of many mRNAs (e.g., see Harrold et al. 1991; Speth and Oberbaumer 1993; Chen et al. 1995). As a result, decay rates obtained in experiments using these agents may not reflect the true stability of the mRNAs. Moreover, many critical mechanistic issues, such as deadenylation kinetics, decay intermediates, and precursor-product relationships cannot be readily addressed by these methods.

In light of these concerns, we have previously reported transcriptional pulsing methods based on the c-fos serum-inducible promoter and the tetracycline-regulated (Tet-off) promoter systems in order to better determine 
mRNA turnover rates in mammalian cells (Shyu et al. 1991; Chen et al. 1994; Xu et al. 1998). Both systems allow quantitation of deadenylation and decay kinetics and elucidation of precursor-product relationships. The c-fos promoter system has been used successfully to determine the mRNA decay kinetics and to identify sequence determinants of several RNA destabilizing elements, such as the AU-rich element (ARE) (Shyu et al. 1991; Chen and Shyu 1994; Chen et al. 1994). Nevertheless, activation of the c-fos promoter requires serum or growth factor stimulation of quiescent cells (Greenberg and Ziff 1984); therefore, this approach is restricted to analysis of mRNA degradation in cells undergoing the $G_{0}$ to $G_{1}$ transition. In addition, most transformed cell lines cannot readily be made quiescent by serum starvation, and the use of serum induction complicates analysis of signaling pathways that may control mRNA turnover. Many of these limitations can be avoided by using the Tet-off promoter transcriptional pulsing approach (e.g., see Winzen et al. 1999; Yamashita et al. 2005). Importantly, no major physiological side effect was detected at the tetracycline level used in this approach.

In this study, we review the application of transcriptional pulsing approaches to the elucidation of mRNA decay pathways and the roles of RNA-destabilizing elements and broaden these strategies to study regulatory aspects of mammalian mRNA turnover. Previous analyses of mRNA stability utilized transcriptional pulsing systems in cells that were either arrested in $G_{0} / G_{1}$ or undergoing proliferation. It was therefore unclear whether or not an mRNA-destabilizing element, such as ARE, functions in other phases of the cell cycle. As a result, little was known about the role of mRNA turnover in control of cell-cycle-dependent gene expression. In the present study, we combine the Tet-off system with known non-cytotoxic cell-cycle inhibitors to test the mRNA-destabilizing function of ARE in human erythroleukemic K562 cells. Our results show that the transcriptional pulsing approach can be adapted to investigate mRNA turnover arrested at various phases of the cell cycle by pharmacological inhibitors.

To facilitate the investigation of mRNA decay pathways and participating enzymes in mammalian cells, we have also developed rigorous but user-friendly protocols for effective transfection of various cell lines. As the optimal conditions for transfection and transcriptional pulsing for different cells and/or under different situations may vary, we tested a variety of reagents and made extensive modifications of the formerly developed transcriptional pulsing protocols to improve the transfection efficiency. These efforts help to provide a guideline for developing optimal protocols using the transcriptional pulsing approach to study mammalian mRNA stability in vivo.

Recent advances in gene expression knockdown via RNA interference (RNAi) in mammalian cells have provided a powerful means to perform reverse genetics to study gene product functions using cultured cells (Dykxhoorn et al.
2003; Meister and Tuschl 2004; Silva et al. 2004). We have developed protocols that simultaneously and effectively transfect small interfering RNA (siRNA) and plasmid DNA. Combining the improved Tet-off promoter transcriptional pulsing approach with siRNA-mediated mRNA knockdown, we have developed a "consecutive siRNA knockdown" protocol that includes transfection with siRNA and DNA plasmids of cells already transfected with siRNA. This repeated transfection with siRNA greatly enhances the knockdown efficiency of the target gene so that the reporter mRNA expressed from the plasmid can be monitored for alterations of its decay due to depletion of the target gene product.

We have also applied the Tet-off transcriptional pulsing system to the study of mRNA turnover in human bronchial epithelial cells, a cell line responsive to stimulation related to allergic inflammation, for example, TNF- $\alpha$ and IL4 (Atasoy et al. 2003). We have established a stable cell line harboring a gene coding for the tetracycline-responsive transcription factor, tTA, using human bronchial epithelial BEAS-2B cells. A series of tests was performed to identify the optimal conditions for transfection and transcriptional pulsing so that kinetics of mRNA decay can be readily monitored in this stable line. The approaches and protocols we report here will broaden the applications of transcription pulsing to study not only the mechanisms of mRNA decay but also their regulation.

\section{RESULTS}

\section{Monitoring mRNA decay in various phases of the cell cycle}

To test the feasibility of applying the Tet-off driven transcriptional pulsing approach to investigate mRNA decay in various phases of the cell cycle, we analyzed the mRNA-destabilizing effectiveness of two types of ARE in mammalian cells arrested at several points in the cell cycle. The experiments used a stable human erythroleukemic cell line (K562 III-2) that expresses the tTA transcription factor to support transcription from the Tet-off promoter system in the absence of tetracycline (Loflin et al. 1999b). A transcriptional pulsing approach was developed that allowed evaluation of ARE-mediated mRNA decay in K562 III-2 cells differentiated along the megakaryocytic or the erythrocytic lineage (Loflin et al. 1999a).

After electroporation of K562 III-2 cells in the presence of plasmids coding for $\beta$-globin genes containing different AREs in the $3^{\prime}$ UTR, cells were maintained in $40 \mathrm{ng} / \mathrm{mL}$ tetracycline for suppression of transcription from the Tetoff promoter, along with various agents known to arrest cells in particular phases of the cell cycle. FACS analysis revealed that proliferating $\mathrm{K} 562$ cells reside mainly in $\mathrm{G}_{1}$ phase and could be effectively blocked at the $\mathrm{G}_{1} / \mathrm{S}$ boundary by aphidicolin (Pedrali-Noy et al. 1980), at the $G_{2} / M$ boundary by nocodazole (Harper 2005), and in M phase 
by colchicine (Fig. 1; Kamech and Seif 1988). The combinations of these cell cycle agents and tetracycline were not cytotoxic, as there were very few apoptotic cells. Twentyfour hours after transfection, a pulse of mRNA synthesis was induced by introducing fresh medium containing the cell cycle agents but not tetracycline for $3 \mathrm{~h}$. Transcription was then abruptly shut off by adding a high concentration of tetracycline $(500 \mathrm{ng} / \mathrm{mL})$. Aliquots were then taken at various time intervals for analysis of deadenylation and decay of cytoplasmic mRNA by Northern blot analysis.

The results (Fig. 2) showed that both the c-fos ARE, a representative for class I AUUUA-containing ARE, and the GM-CSF ARE, a representative for class II AUUUcontaining ARE (Chen and Shyu 1995), function in $G_{1}, G_{2}$, and $M$ phases. Furthermore, deadenylation precedes decay of the mRNA body in all these phases. Thus, ARE-mediated decay can be monitored in K562 cells arrested at various points in the cell cycle by combining the Tet-off driven transcriptional pulsing approach with pharmacological inhibitors.

\section{A Proliferating}
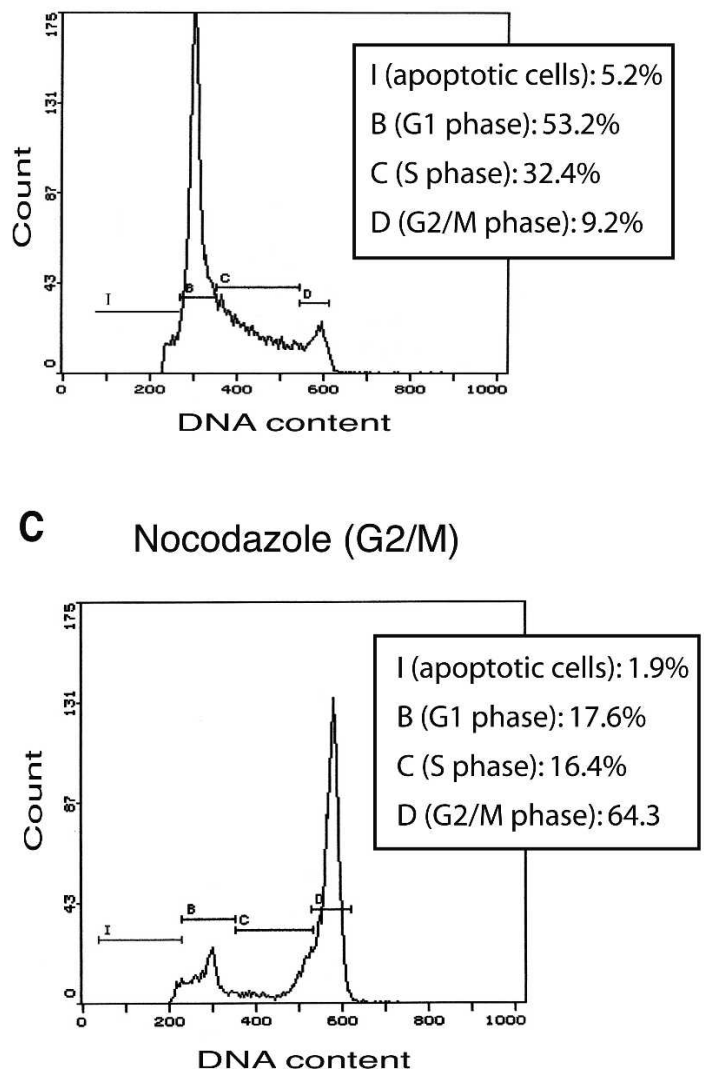

\section{Simultaneous transfection with plasmid DNA and synthetic siRNA}

To facilitate identification of trans-acting factors involved in mRNA decay and elucidate their role in mRNA turnover, we set out to develop protocols to integrate RNAi-mediated gene knockdown with the transcriptional pulsing system. As an effort to simplify the procedure without compromising the transfection efficiency and knockdown effect, we first tried to develop protocols that can simultaneously deliver both the plasmid DNA and the siRNA to the cells effectively. Various transfection reagents were tested because the calcium phosphate-mediated DNA transfection method used in the earlier c-fos serum-inducible promoter and Tet-off promoter protocols is finicky and does not provide sufficient transfection efficiency in many cell lines. Although recent advances in transfection reagents may circumvent this problem, many cannot be used in the presence of antibiotics, a requirement of the Tet-off system. For siRNAmediated knockdown, we tested various transfection reagents

B Aphidicolin (G1/S)

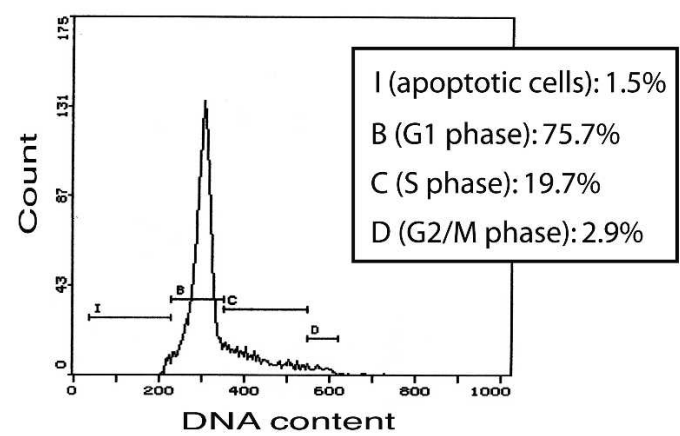

D Colchicine (M)

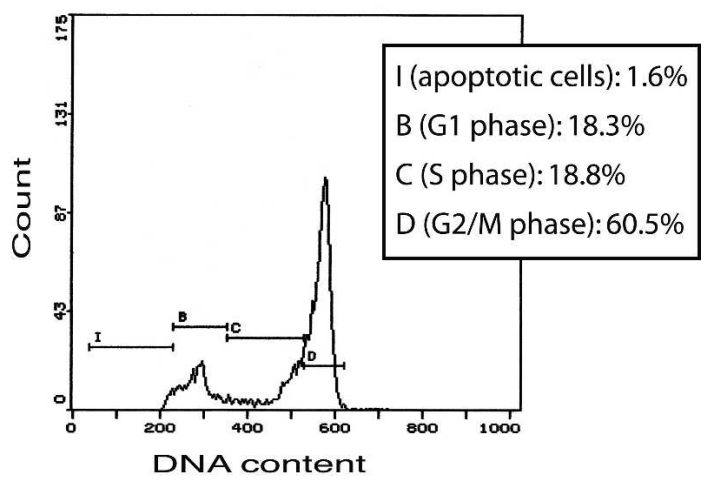

FIGURE 1. FACS analysis of K562III-2 cells arrested using cell cycle blockage reagents. K562 III-2 cells were cultured in the absence (Panel $A$, proliferating) or in the presence of indicated cell cycle inhibitors for $24 \mathrm{~h}$ (Panel $B$, aphidicolin treatment; Panel $C$, nocodazole treatment; Panel $D$, colchicine treatment; see Materials and Methods for treatment detail). Cells were then harvested for fixation followed by flow cytometry to determine the degree of cell synchronization at specific phases. The brackets indicate the cell cycle parameters, with "I" being the population of apoptotic cells (fragmented DNA), "B" being the population of cells in $\mathrm{G}_{1}$ phase, "C" being the population of cells in $S$ phase, and " $D$ " being the population of cells in $\mathrm{G}_{2} / \mathrm{M}$ phases. 


\section{A c-fos ARE}

Proliferating

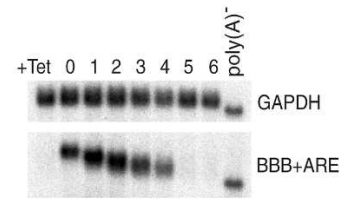

Aphidicolin (G1/S)

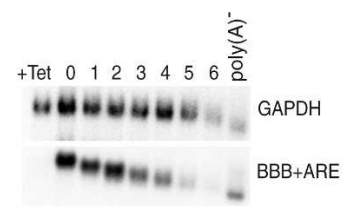

Nocodazole (G2/M)

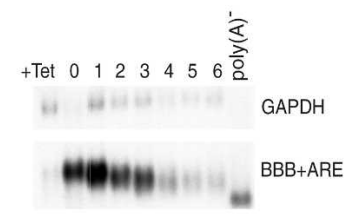

Colchicine (M)
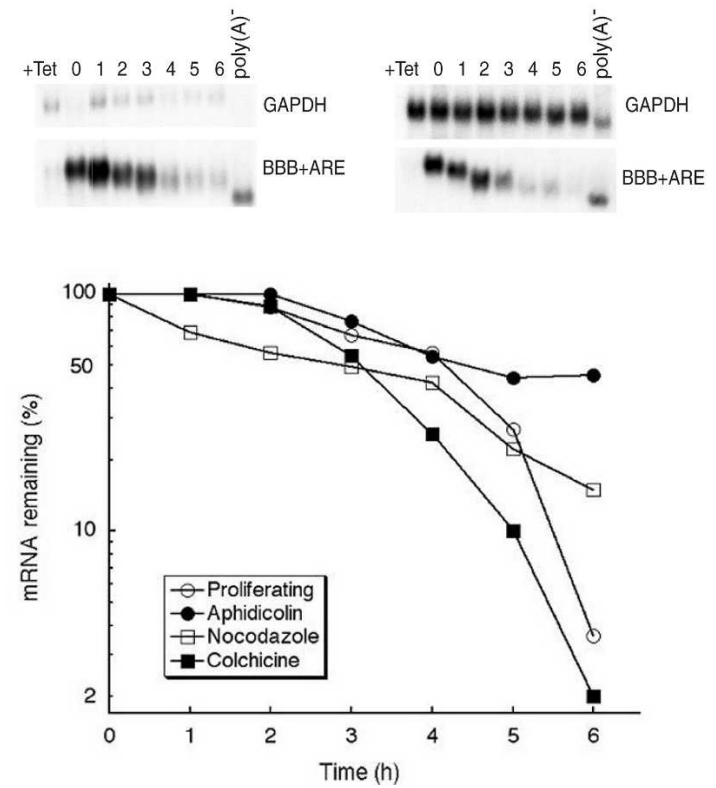

B GM-CSF ARE

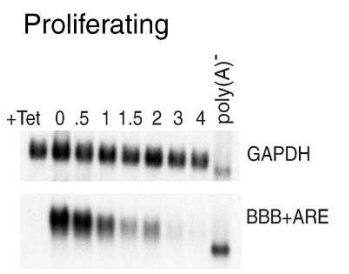

Aphidicolin (G1/S)

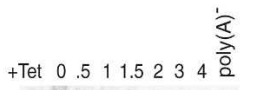

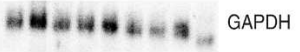

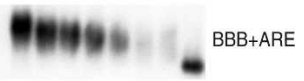

Nocodazole (G2/M)

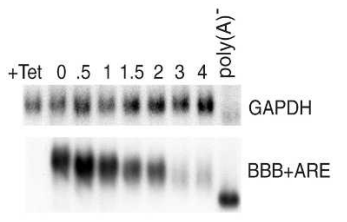

Colchicine (M)
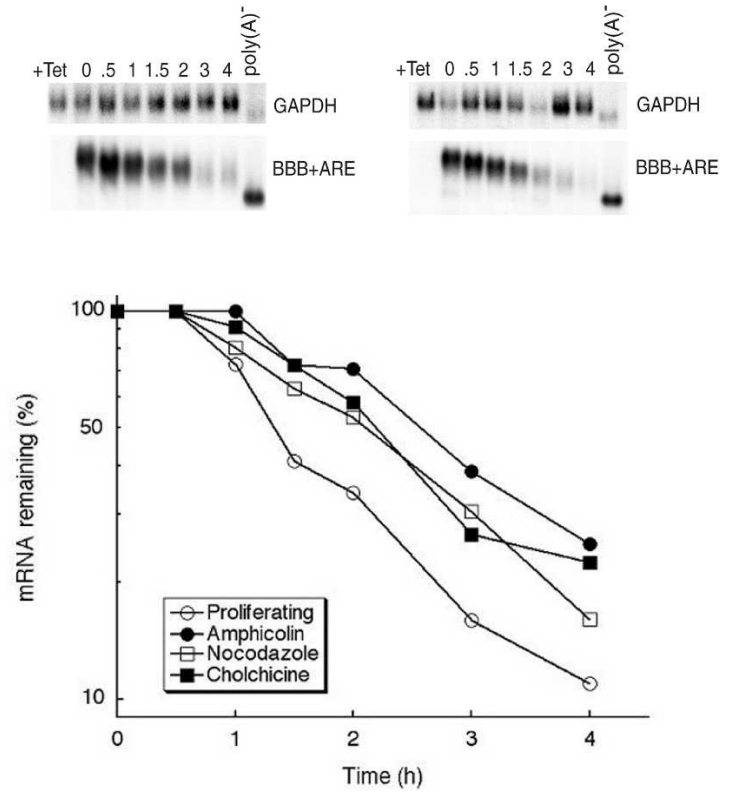

FIGURE 2. The destabilization function of AREs can be monitored at various phases of the cell cycle. K562 III-2 cells were electroporated with pTetBBB+ARE bearing either $(A)$ the c-fos ARE or $(B)$ the GM-CSF ARE. Transfected cells were kept in medium containing $40 \mathrm{ng} / \mathrm{mL}$ tetracycline (Tet) and the indicated cell cycle inhibitor for $24 \mathrm{~h}$ to synchronize cells at the desired phase. Cells were then induced for transcriptional pulsing as described in Materials and Methods. Cytoplasmic RNA was isolated immediately for the zero time point or at various time points after Tet (500 $\mathrm{ng} / \mathrm{mL}$ ) was added as indicated. RNA samples were analyzed by Northern blotting. Poly $(\mathrm{A})^{-}$RNA was prepared in vitro by treating the zero time point sample with oligo (dT) and RNase H. (BBB+ARE) $\beta$-Globin mRNA bearing an ARE; (GAPDH) glyceraldehyde-3-phosphate dehydrogenase mRNA served as an internal control. Semi-log plots were presented in the bottom to show the decay kinetics for BBB+ARE mRNA. Quantification of RNA was obtained by scanning radioactive blots with an imager (Bio-Rad).

and conditions to deliver nonspecific or UNR-specific siRNA into NIH3T3 mouse fibroblasts. UNR is an RNA-binding protein that has been shown to play a key role in mRNA degradation mediated by the c-fos major protein-coding determinant of instability (mCRD) (Triqueneaux et al. 1999; Grosset et al. 2000; Chang et al. 2004). Among the transfection reagents tested, RNAiFect from QIAGEN worked particularly well with NIH3T3 cells, consistently achieving 85\%-95\% knockdown efficiency (Fig. 3). We then tested the feasibility of cotransfecting the cells with both plasmid DNA and siRNA. PolyFect reagent was chosen for introducing DNA into NIH3T3 cells not only because of its high transfection efficiency but also because it tolerates antibiotics, a characteristic required for application of the Tet-off system. The plasmid pcDNA V5-PARN, coding for a mammalian nuclear poly(A) nuclease (Dehlin et al. 2000; Gao et al. 2000; Martinez et al. 2000), was used for this test because the V5-tagged PARN protein expresses well and is readily detected by antibody against the tag. The plasmid DNA was transfected either at the same time as the siRNA or $8 \mathrm{~h}$ later. Our results showed that siRNAs were capable of mediating efficient $(70 \%-80 \%)$ knockdown of UNR in either case (Fig. 3A, lanes 3-5), and that expression levels of V5-PARN protein were higher if the siRNA and the plasmid DNA were transfected simultaneously than when DNA was transfected after siRNA (Fig. 3A, lanes 4,5).

\section{Applying simultaneous DNA-siRNA transfection to the c-fos promoter transcriptional pulsing system}

Combining simultaneous transfection of siRNA and reporter DNA with transcription pulsing systems would allow examination of the effects of decreasing trans-acting factor levels on mRNA decay kinetics. This approach was 
A

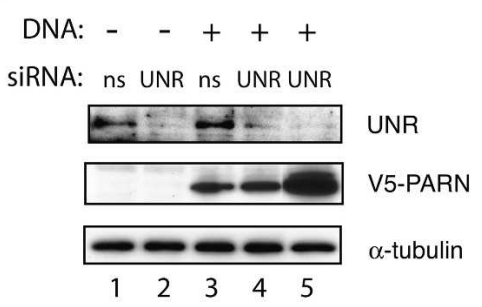

B

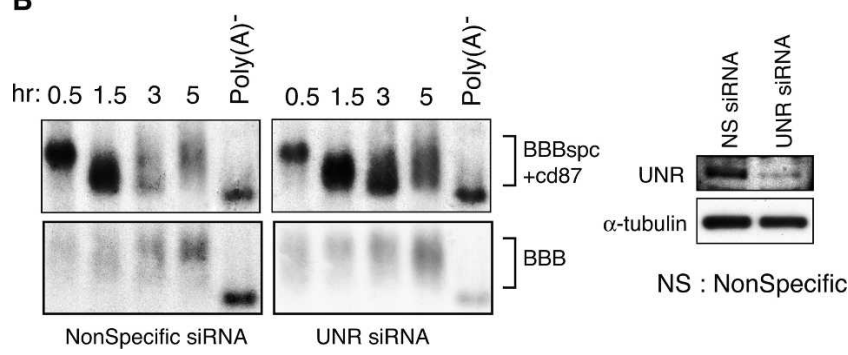

FIGURE 3. DNA and siRNA can be cotransfected effectively without compromising the knockdown efficiency. $(A)$ Western blots of wholecell lysates showing knockdown of UNR protein expression by the specific (UNR) siRNA and not by the nonspecific (ns) siRNA. DNA transfection was carried out at $8 \mathrm{~h}$ after siRNA transfection (lanes 3,4 ) or at the same time (lane 5) as the siRNA was transfected. ( $B$, Left and middle panels) Northern blots showing decay and deadenylation of BBBspc+cd87 mRNA isolated from cells cotransfected with the DNA encoding pBBBspc+cd87 and either nonspecific siRNA (left) or UNRspecific siRNA (middle). The plasmid pSV-BBB, from which $\beta$-globin was expressed constitutively (SV-BBB) and which served as an internal standard, was cotransfected at the same time. (Right panel) Western blots of whole-cell lysate showing knockdown of UNR protein expression by the specific (UNR) siRNA and not by the nonspecific (NS) siRNA.

first tried with the c-fos serum-inducible promoter system to verify the role of UNR in mRNA degradation mediated by the $\mathrm{c}$-fos mCRD. The $\mathrm{pBBBspc}+\mathrm{cd} 87$ plasmid coding for a hybrid $\beta$-globin transcript bearing the $c$-fos $\mathrm{mCRD}$, and a control plasmid, pSV-BBB, encoding a stable $\beta$-globin message that served as an internal standard (Chen and Shyu 1994), were mixed with PolyFect transfection reagent. The siRNA against mouse UNR was prepared with RNAiFect and then added to the cells together with the DNA/ PolyFect mixture. Twenty-one hours later, the cells were serum-starved in DMEM/0.5\% calf serum for $25 \mathrm{~h}$ and then stimulated with DMEM/20\% calf serum. Cell extracts and RNA samples were isolated at the indicated times for Western blot and Northern blot analyses. As shown in Figure 3B, UNR protein expression was significantly decreased by UNR-specific siRNA ( $~ 70 \%$ knockdown efficiency), accompanied by a decreased rate of BBBspc+cd87 mRNA deadenylation and decay, consistent with previous findings (Chang et al. 2004). These results show that investigation of the mRNA decay mechanism and identification of participating factors can be facilitated by combining the simultaneous transfection protocol with the transcriptional pulsing approach.

\section{Optimizing induction time and reporter levels for the Tet-off promoter system using PolyFect reagent}

Applying the approach described above to time-course experiments using the Tet-off promoter driven transcription pulsing system would further facilitate investigation of mRNA decay under a wide variety of physiological conditions in mammalian cells. To optimize conditions for the Tet-off system using the PolyFect reagent, time-course experiments were performed to determine how long a transcriptional pulse is needed to produce a uniformly sized pool of reporter mRNA for study of deadenylation and decay kinetics. NIH3T3 B2A2 cells, which express the tTA transcription factor to support transcription from the Tet-off promoter system in the absence of tetracycline $(\mathrm{Xu}$ et al. 1998), were cultured in medium containing tetracycline $(25-30 \mathrm{ng} / \mathrm{mL})$ for $24 \mathrm{~h}$ before transfection with a plasmid encoding an unstable mRNA, the $\beta$-globin mRNA carrying a nonsense codon (BBB-PTC) that targets the transcript for rapid decay via the nonsense-mediated decay pathway (Lim et al. 1992; Thermann et al. 1998; Chen and Shyu 2003). A DNA/PolyFect mixture was prepared and added to the culture dish as described in Materials and Methods. After $48 \mathrm{~h}$, transfected cells were cultured in fresh medium without tetracycline for various time periods to produce a transcription pulse before transcription was shut down by adding tetracycline $(500 \mathrm{ng} / \mathrm{mL})$ to the medium. RNA was isolated and analyzed by Northern blotting. As shown in Figure 4A, 100-110 min of induction produced a homogeneous pulse of mRNA synthesis when using PolyFect reagent, compared with the 150-min induction used with calcium phosphate transfection (Xu et al. 1998). By $120 \mathrm{~min}$, some mRNA had already undergone deadenylation, giving a somewhat smeared band.

Another critical factor to be considered is the optimal amount of the DNA required for transfection, as the transfection efficiency varies when different reagents are used. While using too little DNA will not produce enough signal for accurate data analysis, introducing too much DNA into a cell can result in excess mRNA synthesis, which may saturate the decay machinery and thus interfere with rapid mRNA decay. Therefore, even though the transfection efficiency with the Polyfect reagent is much higher than that with calcium phosphate, it is crucial to empirically identify the optimal amount of plasmid DNA. We performed time course experiments using different amounts of pTet-BBB-PTC DNA with the Tet-off system and the PolyFect reagent in NIH3T3 B2A2 cells. Using an unstable mRNA as a reporter here allowed us to calibrate the system for rapid decay kinetics. Transcription was pulsed for $110 \mathrm{~min}$ as described above, and the mRNA was analyzed by Northern blot. The results showed that the reporter mRNA was readily detected by Northern blot analysis when a $10-\mathrm{cm}$ dish of NIH3T3 B2A2 cells was transfected with $0.1-0.5 \mu \mathrm{g}$ but not with $0.05 \mu \mathrm{g}$ of reporter plasmid DNA (Fig. 4B). However, rapid decay of BBB-PTC 
A

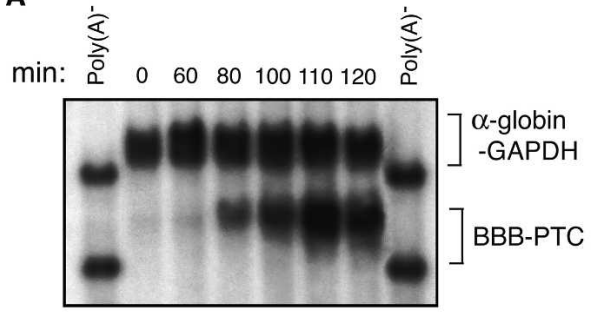

B

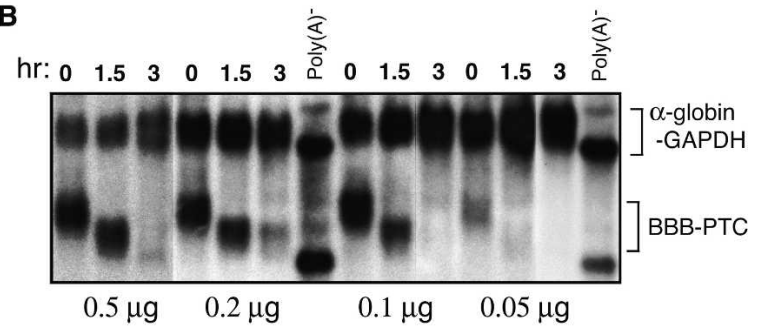

C

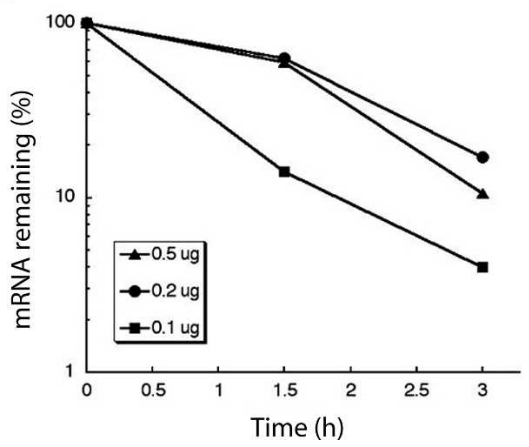

FIGURE 4. Test for the optimal induction time and reporter plasmid levels for the Tet-off promoter system using PolyFect reagent. $(A)$ NIH3T3 B2A2 cells were transfected with $1 \mu \mathrm{g}$ of pTetBBB-PTC and $1 \mu \mathrm{g}$ of pSV $\alpha$-globin/GAPDH in the presence of Tet $(25 \mathrm{ng} / \mathrm{mL})$. Cytoplasmic RNA was isolated at various times after removal of tetracycline as indicated. (B) NIH3T3 B2A2 cells transfected with different amounts of pTetBBB-PTC were transcriptionally pulsed as described in Materials and Methods, and transcription was shut down after $110 \mathrm{~min}$ by adding $500 \mathrm{ng} / \mathrm{mL}$ tetracycline to culture medium. The number above each lane corresponds to hours (hr) after tetracycline addition. $\alpha$-Globin/GAPDH mRNA was expressed constitutively and served as an internal standard for transfection efficiency and sample handling. (C) Semi-log plot showing the decay kinetics for BBB-PTC mRNA. Quantification of RNA was obtained by scanning radioactive blots with an imager (Bio-Rad).

mRNA was retarded when the cells were transfected with 0.2 or $0.5 \mu \mathrm{g}$ of plasmid DNA (Fig. 4B,C).

\section{Analyzing mRNA deadenylation and decay kinetics using the Tet-off system combined with consecutive siRNA knockdown and simultaneous transfection}

The knockdown efficiency of different siRNAs exhibits significant variability. Insufficient knockdown of a target gene may complicate interpretation of experimental results, especially when partial knockdown of the target does not give rise to a phenotype. We therefore developed a "consecutive-knockdown" protocol involving repeated siRNA transfections that significantly increased knockdown efficiency (Fig. 5A). Exploiting the ability to cotransfect plasmid DNA and siRNA, the plasmid DNA was simply included in the second knockdown step. Combining consecutive knockdown with simultaneous transfection of DNA and siRNA provides a new protocol to analyze the impact on mRNA decay of the knockdown of a decay factor. Timecourse experiments were performed using the Tet-off transcription pulsing system combined with repeated siRNA knockdown and simultaneous plasmid DNA/siRNA cotransfection (Fig. 5). In this example, we examined the effect of Dcp2 knockdown in the presence of ectopically expressed mutant Pan2 poly(A) nuclease on decay of BBBPTC mRNA. Mammalian Dcp2 is the catalytic subunit of the Dcpla-Dcp2 decapping complex that removes the $5^{\prime} 7$ methyl guanosine cap from mRNA (Lykke-Andersen 2002; Wang et al. 2002; Piccirillo et al. 2003). Coexpression of a dominant-negative mutant of PAN2 to further block the first phase of deadenylation was necessary to observe an intermediate with a full-length poly(A) tail when Dcp2 expression was knocked down (Chang et al. 2004). NIH3T3 B2A2 cells were split into a $10 \mathrm{~cm}$ dish and transfected with either nonspecific or Dcp2 siRNA. Eight hours later, the cells were trypsinized, recultured to $30 \%$ confluence, and incubated for another $22 \mathrm{~h}$ before simultaneous addition of the siRNA/RNAiFect and DNA/PolyFect mixtures. The DNA/PolyFect mixture combined PolyFect reagent with two plasmid DNAs, one encoding BBB-PTC mRNA and the other coding for a dominant-negative mutant of PAN2 poly(A) nuclease (Pan2mt). Following further incubation for $41 \mathrm{~h}$, a 110 -min transcription pulse was induced from the pTet BBB-PTC construct as described above. At the indicated times, cytoplasmic RNA was extracted for Northern blot analysis, and cytoplasmic lysates were prepared for Western blot analysis of knockdown efficiency. Conventional siRNA/DNA simultaneous transfection, representing "single-knockdown," was performed in parallel as a control; these cells were harvested 43-48 h after siRNA-DNA transfection. As shown in Figure 5A, the levels of Dcp2 protein were reduced more by the consecutive siRNA transfection (Fig. 5A, lane $5,++, \sim 85 \%$ knockdown efficiency) than the control single siRNA transfection (Fig. 5A, lane $4, \sim 50 \%$ knockdown efficiency). The results from Northern blot analysis showed that a decay intermediate with full-length poly(A) tail was observed only with the consecutive knockdown of Dcp2, not with the single knockdown (Fig. 5E,F).

\section{Establishment of a stable BEAS-2B cell line harboring the tetracycline-responsive trans-acting factor for use with the Tet-off transcriptional pulsing system}

To further broaden the application of the transcriptional pulsing strategy, we employed the Tet-off system to study 
A

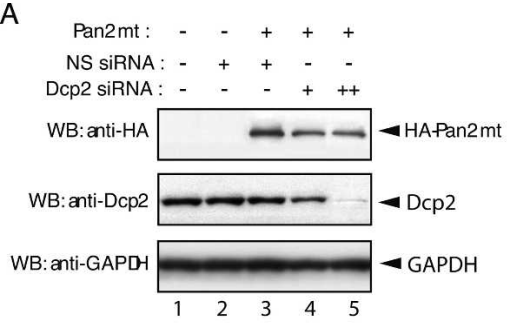

B

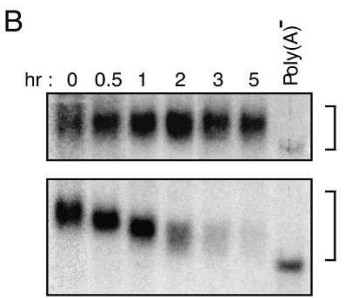

no siRNA

D

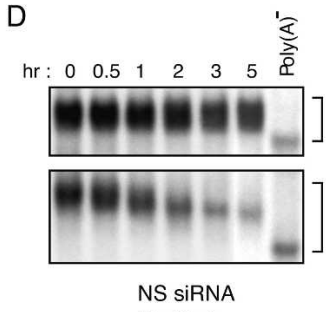

Pan2mt
C

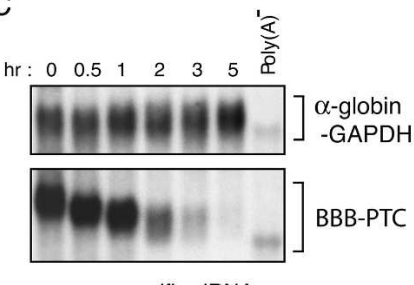

non-specific siRNA

E

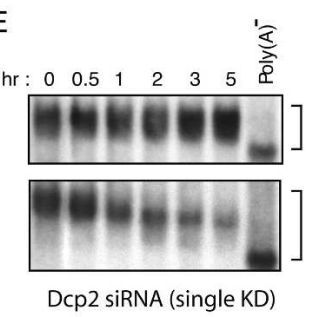

Pan2mt
F

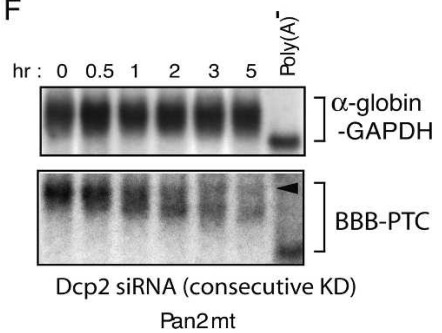

FIGURE 5. A decay intermediate with full-length $\operatorname{poly}(A)$ tail can be detected using the "consecutive-knockdown" approach. $(A)$ Western blot analysis showing the expression of HA-tagged mutant PAN2 and knockdown of DCP2 protein expression in NIH3T3 B2A2 cells ("+" indicates the DNA or siRNA was transfected once, and "++" indicates the siRNA was transfected twice). The blot was probed with antibodies against HA-tag, DCP2, and GAPDH. (B-F) Northern blots showing deadenylation and decay of nonsense codon containing $\beta$-globin (BBB-PTC) mRNA in the $(B, C)$ absence or $(D, F)$ presence of ectopically expressed mutant PAN2, or combined with siRNA-mediated $(E)$ single or $(F)$ consecutive knockdown of DCP2. $\alpha$-Globin/GAPDH mRNA was expressed constitutively and served as an internal standard for transfection efficiency and sample handling.

mRNA decay in human bronchial epithelial BEAS-2B cells (Churchill et al. 1992), which are responsive to stimulation related to allergic inflammation in the airway (e.g., Atsuta et al. 1997; Atasoy et al. 2003). The gene coding for the tetracycline-responsive trans-acting factor, tTA, was stably introduced into the BEAS-2B cell line as described in Materials and Methods. It should be noted that among a few different transfection reagents we have tried, FuGENE 6 reagent (Roche) gives the best transfection efficiency for BEAS-2B cells and also can be used in the presence of tetracycline. The cells were transfected with DNA coding for a luciferase gene driven by the Tet-off promoter, and the clone (BEAS-2B-19) that supports the highest luciferase activity in the absence of tetracycline and also shows the highest repression of luciferase activity in the presence of tetracycline was chosen for further characterization.

Our previous studies (Xu et al. 1998; Loflin et al. 1999b) showed that a successful Tet-off-promoter-driven transcriptional pulse requires a stable tTA-harboring line that exhibits $>99 \%$ inhibition of gene expression from the Tet- off-promoter-driven in the presence of $20-40 \mathrm{ng} / \mathrm{mL}$ tetracycline. This property ensures a short burst of transcription and thus the synthesis of a pool of mRNA homogeneous in size, a necessary prerequisite for monitoring deadenylation and decay kinetics. To test this critical characteristic, BEAS-2B-19 cells were transiently transfected with a plasmid (pTet-flag-eGFP) coding for the enhanced green fluorescent protein (eGFP) driven by the Tet-off promoter in the absence of tetracycline. Immediately following transfection, the cells were cultured for $24 \mathrm{~h}$ in either the absence or in increasing amounts (30, 50 , or $500 \mathrm{ng} / \mathrm{mL}$ ) of tetracycline. Western blot analysis (Fig. 6A) showed that $30 \mathrm{ng} / \mathrm{mL}$ tetracycline is sufficient to repress $>99 \%$ of eGFP protein expression in BEAS-2B-19 cells when compared to the level of eGFP from cells that were cultured without tetracycline.

To develop the Tet-off promoterdriven transcriptional protocol using clone BEAS-2B-19, we then tested the expression of $\beta$-globin mRNA whose synthesis is driven by the Tet-off promoter (Tet-BBB) in BEAS-2B-19 cells in response to the removal of tetracycline. The plasmid pTet-BBB was transiently transfected into BEAS-2B-19 cells using FuGENE 6 reagent in the presence of tetracycline $(30 \mathrm{ng} / \mathrm{mL})$. The kinetics of transcription resumption when the transfected cells were switched from medium containing tetracycline to medium without tetracycline for various time periods was evaluated by Northern blot analysis (Fig. $6 \mathrm{~B})$. The results showed that there is a time lag of $<2 \mathrm{~h}$ prior to the appearance of an intense discrete band corresponding to a significant amount of mRNA with a full-length poly(A) tail. These data indicate that significant transcription was accomplished in BEAS-2B-19 cells between 2 and $3 \mathrm{~h}$ after removal of tetracycline.

Transcriptional pulsing was then utilized to monitor mRNA deadenylation and decay kinetics in BEAS-2B-19 cells. The cells were transfected with pTet-Flag-eGFP, and the deadenylation and decay kinetics of eGFP mRNA were analyzed by Northern blotting (Fig. 6C). The results show that eGFP mRNA undergoes slow and synchronized deadenylation prior to decay of the RNA body. Collectively, these experiments demonstrate the establishment of new mammalian tTA cell lines using our protocol for use in rigorous transcriptional pulsing strategies to study mRNA deadenylation and decay. 
A

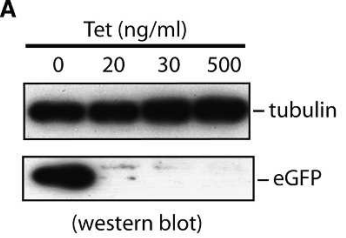

B

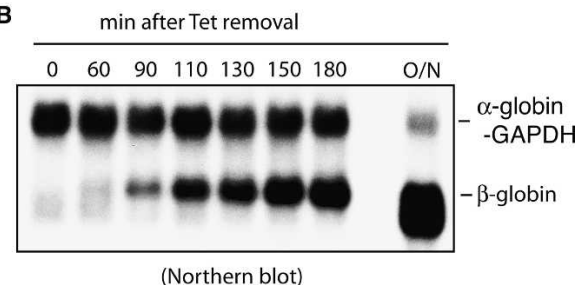

C

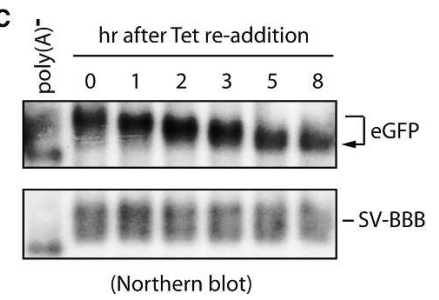

FIGURE 6. Expression kinetics of the Tet-regulated promoter in human bronchial epithelial BEAS-2B cells. (A) Regulation of expression by tetracycline in the human bronchial epithelial BEAS-2B stable line harboring tTA. The BEAS-2B stable clone 19 (BEAS-2B-19) was transfected with pTet-Flag-eGFP. After transfection, cells were split into four dishes and grown for $48 \mathrm{~h}$ in various concentrations of tetracycline (Tet) as indicated. Total cytoplasmic lysates were isolated, and Western blot analysis was performed. Blots were then probed with an anti-Flag antibody for the eGFP and an anti-tubulin antibody for tubulin. $(B)$ Kinetics of transcription resumption when tetracycline is removed. BEAS-2B-19 cells were grown in the presence of tetracycline $(30 \mathrm{ng} / \mathrm{mL})$. At $48 \mathrm{~h}$ after transient cotransfection with plasmids pTet$\mathrm{BBB}$ and $\mathrm{pSV} \alpha 1 / \mathrm{GAPDH}$ (control), expression of the Tet-regulated gene was induced by replacing tetracycline-containing medium with fresh medium without tetracycline. Total cytoplasmic RNA was isolated at the times indicated at the top and subjected to Northern blot analysis. (C) Transcriptional pulsing achieved by modulating tetracycline in the culture medium. Transient mRNA expression from the Tet-regulated Flag-eGFP gene was induced for $110 \mathrm{~min}$ by transferring the cells to fresh culture medium without tetracycline, followed by addition of $500 \mathrm{ng} / \mathrm{mL}$ tetracycline to the medium to block transcription for the indicated time intervals to monitor mRNA decay analyzed by Northern blotting. In this blot, $\beta$-globin mRNA (SV-BBB) constitutively transcribed from cotransfected plasmid pSVBBB served as an internal standard.

\section{DISCUSSION}

In this study, we expand the application of transcriptional pulsing to study mammalian mRNA decay in tissue culture cells arrested at various phases of the cell cycle by pharmacological inhibitors. The results demonstrate that both the c-fos and GM-CSF AREs can direct rapid mRNA degradation in the $G_{1}, G_{1} / S, M$, and $M / G_{2}$ phases of the cell cycle in human erythroleukemic K562 cells. Taken together with results from cells in $G_{0}$ (Xu et al. 1998), the results suggest that the destabilizing function of these AREs is cell cycle independent. The present data also show that dead- enylation precedes decay of the mRNA body regardless of the cell cycle phase (Fig. 2), providing additional evidence that deadenylation is a key step triggering mammalian mRNA decay (Muhlemann 2005). One important implication is that an ARE partnered with cell-cycle-dependent transcription regulation could be used to tightly control the levels of a cell-cycle-regulated transcript by quickly destabilizing the transcript following transcription shut-off. It will be interesting to learn whether the same ARE-binding protein is used to target a cognate transcript for rapid degradation in different phases of the cell cycle. In addition, the protocol can also be employed to study mechanisms underlying regulation of cell-cycle-related mRNAs, such as cyclin mRNA (Wang et al. 2000), at the level of mRNA stability.

The consecutive-knockdown siRNA protocol increases the knockdown efficiency to $85 \%$ from the $50 \%$ observed with a single siRNA treatment (Fig. 5A). Plasmid DNAs coding for a reporter mRNA and/or a decay factor can be conveniently cotransfected with the second dose of siRNA. One advantage of the present approach is that variations in plasmid transfection efficiency have little effect on changes in mRNA decay kinetics caused by specific knockdown of decay factors. This is because the gene targeted by siRNA is already effectively knocked down before the reporter mRNA is expressed from plasmid DNA. With effective knockdown of the target decay factor, this protocol combined with a transcription pulsing approach makes it possible to monitor changes in deadenylation and decay kinetics and to trap decay intermediates that reveal limiting steps during mRNA decay. This approach is particularly useful when there are multiple pathways for degrading mRNA. As demonstrated in Figure 5, a decay intermediate with a full-length poly(A) tail was only detected when ectopic expression of an inactive PAN2 mutant was combined with effective DCP2 knockdown using the consecutive-knockdown protocol.

Several experimental details are important for success of the modified transcriptional pulsing strategy for measuring poly(A) shortening and mRNA decay kinetics and identifying the participating enzymes. First, the amount of transfected plasmid DNA coding for the reporter mRNA must be empirically optimized with a positive control coding for an unstable message to avoid saturation of the decay machinery with excess mRNA. Second, the length of the transcriptional pulse must be optimized empirically for the transient transfection reagent used. As shown in Figure $4 \mathrm{~A}, 100-110 \mathrm{~min}$ of induction time gave the best pool of synchronized mRNA molecules when PolyFect was used for transfecting NIH3T3 cells, whereas a 150-min induction time worked well for calcium phosphate-based transfection (Xu et al. 1998). This is particularly relevant because a prolonged transcription pulse would produce artifactually slow deadenylation kinetics, as deadenylation would already be largely completed. 
Previously we have employed the transcriptional pulsing approach to determine mRNA deadenylation and decay kinetics in two different tTA-harboring stable lines, NIH3T3-B2A2 cells and K562 III-2 cells (Xu et al. 1998; Loflin et al. 1999a; Yamashita et al. 2005). In the present study, the approach was successfully applied to a different differentiated cell line. We established a new tTA stable line, BEAS-2B-19, using human bronchial epithelial cells. After testing various reagents and conditions, we have found that FuGENE 6 gives an effective transfection when a $10-\mathrm{cm}$ dish of BEAS-2B cells was transfected with $10 \mathrm{ng}$ of reporter plasmid DNA (data not shown). It is striking that transcriptional pulsing kinetics detected using the BEAS-2B-19 stable line (Fig. 6) directly parallel the results obtained previously using NIH3T3 B2A2 and K562 III-2 cell lines (Xu et al. 1998; Loflin et al. 1999b). A population of mRNA homogeneous in size appears in the cytoplasm following removal of tetracycline for $\sim 110 \mathrm{~min}$, making it possible to study deadenylation and decay kinetics and precursorproduct relationships during mRNA decay. Levels of tetracycline sufficient to repress $>99 \%$ of mRNA expression from the Tet-off promoter-driven gene in these cells are also in a similar range $(30-40 \mathrm{ng} / \mathrm{mL})$. Thus, the Tet-off transcriptional pulsing system has been successfully applied to three different mammalian tTA stable cell lines. Human erythroleukemic K562 cells were grown as a suspension, and human bronchial epithelial cells and mouse NIH3T3 fibroblasts were grown as a monolayer. In all three cases, mRNA deadenylation kinetics and the decay precursorproduct relationships can be readily followed by Northern blot analysis without using transcription inhibitors, such as actinomycin D and DRB. The results demonstrate the generality of our approach for developing protocols and strategies to optimize the experimental conditions, which has further broadened the application of the transcription pulsing systems.

In summary, we have successfully combined the consecutive-knockdown strategy with simultaneous transfection of plasmid DNA and siRNA and the transcription pulsing systems to decipher mechanistic steps in mammalian mRNA turnover (e.g., see Xu et al. 1998; Loflin et al. 1999a; Yamashita et al. 2005). The methods we describe here have simplified procedures for transfecting multiple nucleic acids into cultured mammalian cells via distinct transfection reagents and allow development of a more rigorous method to identify and characterize trans-acting factors involved in mammalian mRNA decay. Using the information reported here, the applications of transcription pulsing systems can be further expanded to study not only the mechanisms of mRNA decay but also their regulation. For instance, recent findings that microRNA (miRNA)mediated mRNA decay also starts with deadenylation (Giraldez et al. 2006; Wu et al. 2006) indicate that the transcriptional pulsing approach, which can readily address many critical mechanistic issues including deadenylation kinetics, will facilitate our understanding of how miRNAs tie into the mRNA decay machinery to accomplish gene silencing in mammalian cells. In addition, with the protocol developed to study mRNA decay in cells arrested at individual phases of the cell cycle, the role of miRNAs in controlling mRNA decay during the cell cycle can also be studied. Our approaches to optimize these protocols provide guidelines for more widespread development of new protocols using different cells to study mRNA turnover under various physiological conditions.

\section{MATERIALS AND METHODS}

\section{siRNA and plasmid construction}

Construction of the plasmids was described previously:

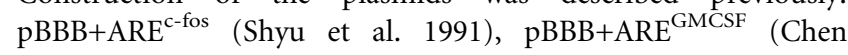
et al. 1995), pSVBBB (Shyu et al. 1989), pSV $\alpha$-globin/GAPDH (Chen and Shyu 1994), pBBBspc+cd87 (Grosset et al. 2000), pTet BBB-PTC (Chen and Shyu 2003), pTet-BBB+ARE (Xu et al. 1998), pcDNA3.1/GS-PARN and pSR-HA-PAN2-mt (Chang et al. 2004), and pT18 (Shyu et al. 1989). The plasmid pTet-Flag-eGFP was created as follows. The myc-tag was first replaced with the Flag-tag from the plasmid pTet-Myc-over to create pTet-Flagover. A PCR-amplified fragment spanning the open reading frame of eGFP was then subcloned immediately downstream from the Flag-tag to create the pTet-Flag-eGFP. The final construct was confirmed by DNA sequencing to ensure in-frame fusion. The siRNAs targeting mouse UNR (accession. no. NM_144901) and mouse Dcp2 (accession. no. BAB30946) and the nonspecific control siRNAs were purchased from Dharmacon (SMARTpool).

\section{Cell synchronization and flow cytometry}

K562 III-2 cells were maintained as a suspension in RPMI 1640 with $10 \%$ fetal bovine serum and $500 \mathrm{ng} / \mathrm{mL}$ tetracycline at $37^{\circ} \mathrm{C}$ in $8 \% \mathrm{CO}_{2}$. Cells enriched in the desired phases were obtained by culturing them for $24 \mathrm{~h}$ in the presence of $2.5 \mu \mathrm{g} / \mathrm{mL}$ aphidicolin for $\mathrm{G}_{1} / \mathrm{S}$ phases, $40 \mathrm{ng} / \mathrm{mL}$ nocodazole for $\mathrm{G}_{2} / \mathrm{M}$ phases, or $1 \mathrm{mM}$ colchicines for $\mathrm{M}$ phases. The cells were fixed in methanol followed by RNase treatment and $10 \mu \mathrm{g} / \mathrm{mL}$ propidium iodide staining of DNA. FACS analysis for DNA content was accomplished using a flow cytometer with an excitation wavelength of $420 \mathrm{~nm}$.

\section{Analysis of mRNA deadenylation and decay in $K 562$ cells}

Cell culture, DNA transfection, isolation of total cytoplasmic RNA, and Northern blot analysis were conducted as described previously (Shyu et al. 1996; Loflin et al. 1999b). Briefly, K562 III-2 cells were cultured to a density of $10^{6}$ cells $/ \mathrm{mL}$, harvested, washed with PBS, and resuspended in PRMI 1640 at a density of $7.5 \times 10^{7}$ cells $/ \mathrm{mL}$. Transfection of cells with $50 \mu \mathrm{g}$ of plasmid DNA by electroporation was accomplished in a 2-mm gap cuvette (3-5 $\times 10^{7}$ cells $\left./ 0.4 \mathrm{~mL}\right)$. After electroporation, cells were resuspended in medium containing $40 \mathrm{ng} / \mathrm{mL}$ tetracycline with or without the appropriate cell cycle inhibitor for $24 \mathrm{~h}$. Aliquots $\left(3 \times 10^{6}\right.$ cells $)$ were washed with PBS and re-suspended in 
medium without tetracycline but with cell cycle inhibitor for $3 \mathrm{~h}$ to induce a transcriptional pulse from the Tet-off promoterdriven construct, $\mathrm{pTet}-\mathrm{BBB}+\mathrm{ARE}$. Cytoplasmic RNA was extracted for the zero time point. Alternatively, $500 \mathrm{ng} / \mathrm{mL}$ tetracycline was immediately added back to the cell suspension for the indicated times, and then cytoplasmic RNA was extracted. Northern blot analysis was performed as described previously (Shyu et al. 1996) using a DNA probe spanning 80 bp of the rabbit $\beta$-globin $3^{\prime}$-UTR for detection of the transfected $\beta$-globin message containing an ARE (BBB+ARE) without cross-detection of endogenous human $\alpha$-globin mRNA. Note that human K562 erythroleukemic cells do not express $\beta$-globin mRNA. A 300-bp DNA fragment spanning the second exon of GAPDH was labeled for detection of endogenous GAPDH mRNA, as an internal control for variation due to sample handling and transfection efficiency.

\section{Culturing and transfecting NIH3T3 cells}

NIH3T3 cells were cultured in DMEM medium containing 10\% calf serum, $2 \mathrm{mM}$ glutamine, $100 \mathrm{U} / \mathrm{mL}$ penicillin, and $100 \mathrm{mg} /$ $\mathrm{mL}$ streptomycin at $37^{\circ} \mathrm{C}$ and $8 \% \mathrm{CO}_{2}$. For transfection of DNA driven by the c-fos serum-inducible promoter, NIH3T3 cells were split to a density of $1.8 \times 10^{6} / 10$-cm dish $24 \mathrm{~h}$ before transfection. For siRNA transfection, $30 \mu \mathrm{g}$ of siRNAs was diluted into $1 \mathrm{~mL}$ of serum-free medium, and $90 \mu \mathrm{L}$ of RNAiFect (QIAGEN) was added and mixed well. For DNA transfection, $7 \mu \mathrm{g}$ of DNA (1.5 $\mu \mathrm{g}$ of test plasmid, $3.0-4.5 \mu \mathrm{g}$ of internal control plasmid pSVBBB, and 1.0-2.5 $\mu \mathrm{g}$ of carrier plasmid pT18) was diluted into $1 \mathrm{~mL}$ of DMEM medium containing $10 \%$ calf serum, and $40 \mu \mathrm{L}$ of PolyFect (QIAGEN) was added and mixed well. Both mixtures were then added simultaneously to the culture dish. For serum induction, after $21 \mathrm{~h}$, cells were serum-starved in DMEM/ $0.5 \%$ calf serum for $25 \mathrm{~h}$ and then stimulated with DMEM/20\% calf serum.

For the time-course experiments using the Tet-off promoterdriven transcription pulsing system, $1.8 \times 10^{6} \mathrm{NIH} 3 \mathrm{~T} 3 \mathrm{~B} 2 \mathrm{~A} 2$ cells (30\% confluence) were plated per $10-\mathrm{cm}$ plate the day before transfection. Seven micrograms of plasmid DNAs $(0.05-2 \mu \mathrm{g}$ of reporter plasmid pTet-BBB-PTC, $1 \mu \mathrm{g}$ of internal control plasmid $\mathrm{pSV} \alpha$-globin/GAPDH, and 4-5.95 $\mu \mathrm{g}$ of carrier plasmid pT18) were transfected using $40 \mu \mathrm{L}$ of PolyFect (QIAGEN). Forty-one hours after transfection, cells were cultured in fresh medium without tetracycline to get a transcription pulse of either $150 \mathrm{~min}$ or $110 \mathrm{~min}$. Transcription was terminated by adding tetracycline to a final concentration of $500 \mathrm{ng} / \mathrm{mL}$ of culture medium. For transfection involving both siRNA and DNA, $90 \mu \mathrm{L}$ of RNAiFect (QIAGEN) mixed with $30 \mu \mathrm{g}$ of siRNA (Dharmacon) and $40 \mu \mathrm{L}$ of PolyFect (QIAGEN) mixed with $7 \mu \mathrm{g}$ of DNA $(0.11 \mu \mathrm{g}$ of reporter plasmid pTet-BBB-PTC, $0.22 \mu \mathrm{g}$ of internal control plasmid pSV $\alpha$-globin-GAPDH, and $6.67 \mu \mathrm{g}$ of either carrier plasmid pT18 or the mutant PAN2 expression vector pSR-HAPAN2-mt) were prepared separately and then added simultaneously to the $10-\mathrm{cm}$ culture dish. For consecutive knockdown, cells were first split to $10-\mathrm{cm}$ dishes and then transfected with $30 \mu \mathrm{g}$ of siRNA using $90 \mu \mathrm{L}$ of RNAiFect. Eight hours later, cells were trypsinized, re-cultured in $10-\mathrm{cm}$ dishes to $30 \%$ confluence, and incubated for another $22 \mathrm{~h}$ before the siRNA/RNAiFect mixture and the DNA/PolyFect mixture (prepared and transfected as described above) were added simultaneously to the culture dish.

\section{Establishment of the BEAS-2B stable cell line expressing the tTA gene}

The human bronchial epithelial cell line, BEAS-2B, was purchased from ATCC and was maintained in F12/DMEM (Invitrogen) containing 5\% heat-inactivated FCS, $2 \mathrm{mM}$ L-glutamine, penicillin $(100 \mathrm{U} / \mathrm{mL})$, and streptomycin $(100 \mathrm{mg} / \mathrm{mL})$. Individual, and not pooled, stable BEAS-2B transfectants of pUHD15-1 encoding tTA, Tet-regulated transactivator, were obtained as described previously except that the cells were transfected using FuGENE 6 reagent (Roche) (Xu et al. 1998; Loflin et al. 1999b). The individual transfectants harboring the tTA plasmid, pUHD15-1, were first screened for tTA activity by transiently transfecting them with pUHC13-1 encoding the firefly luciferase gene driven by the Tet-off promoter. Luciferase activity was analyzed according to the manufacturer's instructions (Promega). The clone showing the highest luciferase activity in the absence of tetracycline and also the highest repression of luciferase activity by tetracycline was chosen for further characterization as described in the Results section.

\section{Preparation of RNA samples and Northern blot analysis}

Cytoplasmic RNA samples were isolated at various time points as described in each case using RNeasy RNA preparation kit (QIAGEN) and analyzed by Northern blotting. Preparation of probes and Northern blot analyses were conducted as described previously (Shyu et al. 1996). RNase H treatment of cytoplasmic mRNA to generate poly(A) ${ }^{-}$RNA and analysis of deadenylation and decay curves were carried out as described previously (Shyu et al. 1991).

\section{Western blot analysis}

Cell lysates were prepared as described previously (Peng et al. 1998), resolved on a 10\% SDS-polyacrylamide gel, and transferred to PVDF membrane (Millipore). The PVDF blots were probed with specific antibodies as described below and detected with SuperSignal West Pico Chemiluminescent Substrate (Pierce). The $\mathrm{mAb}$ (monoclonal antibody) against $\alpha$-tubulin (DM1A; Sigma) was used at 1:5000 to 1:10000 dilution. To detect endogenous UNR in NIH3T3 cells, a polyclonal antibody was generated in rabbits (Chang et al. 2004) and used at 1:2000 dilution. A monoclonal antibody specific for the V5 epitope-tag (Invitrogen) was used at 1:3000 dilution to detect V5-PARN. A monoclonal antibody specific for the HA-tag (Roche) was used at 1:2000 dilution. To detect GAPDH, which served as an internal control for total protein extracts, the GAPDH mAb (Jacson Immunology) was used at 1:5000 dilution. Endogenous mouse DCP2 was detected by a rabbit polyclonal antibody (1:500 dilution) against human DCP2 (kindly provided by M. Kiledjian). To detect Flagtagged eGFP, the anti-Flag antibody (Sigma) was used at 1:500 dilution.

\section{ACKNOWLEDGMENTS}

We thank R. Kulmacz and J. Lever for critical reading of the manuscript, M. Kiledjian for anti-hDCP2 antiserum, and P. Loflin and Y. Zhai for technical assistance. This work was supported by 
the National Institutes of Health (GM 46454) and in part by the Houston Endowment, Inc. and by the Sandler Program for Asthma Research to A.-B.S.

Received May 29, 2007; accepted July 23, 2007.

\section{REFERENCES}

Atasoy, U., Curry, S.L., Lopez de Silanes, I., Shyu, A.-B., Casolaro, V., Gorospe, M., and Stellato, C. 2003. Regulation of eotaxin gene expression by TNF- $\alpha$ and IL-4 through mRNA stabilization: Involvement of the RNA-binding protein HuR. J. Immunol. 171: 4369-4378.

Atsuta, J., Sterbinsky, S.A., Plitt, J., Schwiebert, L.M., Bochner, B.S., and Schleimer, R.P. 1997. Phenotyping and cytokine regulation of the BEAS-2B human bronchial epithelial cell: Demonstration of inducible expression of the adhesion molecules VCAM-1 and ICAM-1. Am. J. Respir. Cell Mol. Biol. 17: 571-582.

Belasco, J.G. and Greenberg, M.E. 1993. Control of messenger RNA stability. In Control of messenger RNA stability (eds. J.G. Belasco and G. Brawerman), pp. 199-210. Academic Press, San Diego.

Chang, T.-C., Yamashita, A., Chen, C.-Y.A., Yamashita, Y., Zhu, W., Durdan, S., Kahvejian, A., Sonenberg, N., and Shyu, A.-B. 2004. UNR, a new partner of poly(A)-binding protein, plays a key role in translationally coupled mRNA turnover mediated by the c-fos major coding-region determinant. Genes \& Dev. 18: 2010-2023.

Chen, C.Y. and Shyu, A.B. 1994. Selective degradation of earlyresponse-gene mRNAs: Functional analyses of sequence features of the AU-rich elements. Mol. Cell. Biol. 14: 8471-8482.

Chen, C.Y. and Shyu, A.B. 1995. AU-rich elements: Characterization and importance in mRNA degradation. Trends Biochem. Sci. 20: $465-470$.

Chen, C.Y. and Shyu, A.B. 2003. Rapid deadenylation triggered by a nonsense codon precedes decay of the RNA body in a mammalian cytoplasmic nonsense-mediated decay pathway. Mol. Cell. Biol. 23: 4805-4813.

Chen, C.Y., Chen, T.M., and Shyu, A.B. 1994. Interplay of two functionally and structurally distinct domains of the c-fos AU-rich element specifies its mRNA-destabilizing function. Mol. Cell. Biol. 14: $416-426$.

Chen, C.Y., Xu, N., and Shyu, A.B. 1995. mRNA decay mediated by two distinct AU-rich elements from c-fos and granulocyte-macrophage colony-stimulating factor transcripts: Different deadenylation kinetics and uncoupling from translation. Mol. Cell. Biol. 15: 5777-5788.

Churchill, L., Friedman, B., Schleimer, R.P., and Proud, D. 1992. Production of granulocyte-macrophage colony-stimulating factor by cultured human tracheal epithelial cells. Immunology 75: 189195.

Dehlin, E., Wormington, M., Korner, C.G., and Wahle, E. 2000. Capdependent deadenylation of mRNA. EMBO J. 19: 1079-1086.

Dykxhoorn, D.M., Novina, C.D., and Sharp, P.A. 2003. Killing the messenger: Short RNAs that silence gene expression. Nat. Rev. Mol. Cell Biol. 4: 457-467.

Gao, M., Fritz, D.T., Ford, L.P., and Wilusz, J. 2000. Interaction between a poly(A)-specific ribonuclease and the $5^{\prime}$ cap influences mRNA deadenylation rates in vitro. Mol. Cell 5: 479-488.

Giraldez, A.J., Mishima, Y., Rihel, J., Grocock, R.J., Van Dongen, S., Inoue, K., Enright, A.J., and Schier, A.F. 2006. Zebrafish MiR-430 promotes deadenylation and clearance of maternal mRNAs. Science 312: 75-79.

Greenberg, M.E. and Ziff, E. 1984. Stimulation of 3T3 cells induces transcription of the c-fos proto-oncogene. Nature 311: 433-438.

Grosset, C., Chen, C.-Y.A., Xu, N., Sonenberg, N., JacqueminSablon, H., and Shyu, A.-B. 2000. A mechanism for translationally coupled mRNA turnover: Interaction between the poly(A) tail and a c-fos RNA coding determinant via a protein complex. Cell 103: 29-40.

Harper, J.V. 2005. Synchronization of cell populations in $G_{1} / S$ and $\mathrm{G}_{2} / \mathrm{M}$ phases of the cell cycle. Methods Mol. Biol. 296: 157-166.

Harrold, S., Genovese, C., Kobrin, B., Morrison, S.L., and Milcarek, C. 1991. A comparison of apparent mRNA half-life using kinetic labeling techniques vs decay following administration of transcriptional inhibitors. Anal. Biochem. 198: 19-29.

Kamech, N. and Seif, R. 1988. Effect of microtubule disorganizing or overstabilizing drugs on the proliferation of rat 3T3 cells and their virally induced transformed derivatives. Cancer Res. 48: 48924896.

Lim, S., Sigmund, C., Gross, K., and Maquat, L. 1992. Nonsense codons in human $\beta$-globin mRNA result in the production of mRNA degradation products. Mol. Cell. Biol. 12: 1149-1161.

Loflin, P.T., Chen, C.-Y.A., and Shyu, A.-B. 1999a. Unraveling a cytoplasmic role for hnRNP D in the in vivo mRNA destabilization directed by the AU-rich element. Genes \& Dev. 13: 18841897.

Loflin, P.T., Chen, C.-Y.A., Xu, N., and Shyu, A.-B. 1999b. Transcriptional pulsing approaches for analysis of mRNA turnover in mammalian cells. Methods 17: 11-20.

Lykke-Andersen, J. 2002. Identification of a human decapping complex associated with hUpf proteins in nonsense-mediated decay. Mol. Cell. Biol. 22: 8114-8121.

Martinez, J., Ren, Y., Thuresson, A., Hellman, U., Astrom, J., and Virtanen, A. 2000. A 54-kDa fragment of the poly(A)-specific ribonuclease is an oligomeric, processive, and cap-interacting poly(A)-specific 3' exonuclease. J. Biol. Chem. 275: 24222-24230.

Meister, G. and Tuschl, T. 2004. Mechanisms of gene silencing by double-stranded RNA. Nature 431: 343-349.

Meyer, S., Temme, C., and Wahle, E. 2004. Messenger RNA turnover in eukaryotes: Pathways and enzymes. Crit. Rev. Biochem. Mol. Biol. 39: 197-216.

Muhlemann, O. 2005. Applying the brakes on gene expression. Nat. Struct. Mol. Biol. 12: 1024-1025.

Parker, R. and Song, H. 2004. The enzymes and control of eukaryotic mRNA turnover. Nat. Struct. Mol. Biol. 11: 121-127.

Pedrali-Noy, G., Spadari, S., Miller-Faures, A., Miller, A.O., Kruppa, J., and Koch, G. 1980. Synchronization of HeLa cell cultures by inhibition of DNA polymerase $\alpha$ with aphidicolin. Nucleic Acids Res. 8: 377-387.

Peng, S.-S., Chen, C.-Y.A., Xu, N., and Shyu, A.-B. 1998. RNA stabilization by the AU-rich element binding protein, HuR, an ELAV protein. EMBO J. 17: 3461-3470.

Piccirillo, C., Khanna, R., and Kiledjian, M. 2003. Functional characterization of the mammalian mRNA decapping enzyme hDcp2. RNA 9: 1138-1147.

Ross, J. 1995. mRNA stability in mammalian cells. Microbiol. Rev. 59: 423-450.

Shyu, A.-B., Greenberg, M.E., and Belasco, J.G. 1989. The c-fos mRNA is targeted for rapid decay by two distinct mRNA degradation pathways. Genes \& Dev. 3: 60-72.

Shyu, A.-B., Belasco, J.G., and Greenberg, M.G. 1991. Two distinct destabilizing elements in the c-fos message trigger deadenylation as a first step in rapid mRNA decay. Genes \& Dev. 5: 221-232.

Shyu, A.-B., Garcia-Sanz, J.A., and Mullner, E. 1996. Analysis of mRNA decay in mammalian cells. In The immunology methods manual (ed. I. Lefkovits), pp. 450-462. Academic Press, London.

Silva, J., Chang, K., Hannon, G.J., and Rivas, F.V. 2004. RNAinterference-based functional genomics in mammalian cells: Reverse genetics coming of age. Oncogene 23: 8401-8409.

Speth, C. and Oberbaumer, I. 1993. Expression of basement membrane proteins: Evidence for complex post-transcriptional control mechanisms. Exp. Cell Res. 204: 302-310.

Thermann, R., Neu-Yilik, G., Deters, A., Frede, U., Wehr, K., Hagemeier, C., Hentze, M.W., and Kulozik, A.E. 1998. Binary specification of nonsense codons by splicing and cytoplasmic translation. EMBO J. 17: 3484-3494. 


\section{Chen et al.}

Triqueneaux, G., Velten, M., Franzon, P., Dautry, F., and JacqueminSablon, H. 1999. RNA binding specificity of Unr, a protein with five cold shock domains. Nucleic Acids Res. 27: 1926-1934.

Wang, W., Caldwell, M.C., Lin, S., Furneaux, H., and Gorospe, M. 2000. HuR regulates cyclin A and cyclin B1 mRNA stability during cell proliferation. EMBO J. 19: 2340-2350.

Wang, Z., Jiao, X., Carr-Schmid, A., and Kiledjian, M. 2002. The hDcp2 protein is a mammalian mRNA decapping enzyme. Proc. Natl. Acad. Sci. 99: 12663-12668.

Wilusz, C.W., Wormington, M., and Peltz, S.W. 2001. The cap-to-tail guide to mRNA turnover. Nat. Rev. Mol. Cell Biol. 2: 237-246.

Winzen, R., Kracht, M., Ritter, B., Wilhelm, A., Chen, C.-Y.A., Shyu, A.-B., Muller, M., Gaestel, M., Resch, K., and
Holtmann, H. 1999. The p38 MAP kinase pathway signals for cytokine-induced mRNA stabilization via MAP kinase-activated protein kinase 2 and an AU-rich region-targeted mechanism. EMBO J. 18: 4969-4980.

Wu, L., Fan, J., and Belasco, J.G. 2006. MicroRNAs direct rapid deadenylation of mRNA. Proc. Natl. Acad. Sci. 103: 4034-4039.

Xu, N., Loflin, P., Chen, C.-Y.A., and Shyu, A.-B. 1998. A broader role for AU-rich element-mediated mRNA turnover revealed by a new transcriptional pulse strategy. Nucleic Acids Res. 26: 558-565.

Yamashita, A., Chang, T.C., Yamashita, Y., Zhu, W., Zhong, Z., Chen, C.Y., and Shyu, A.B. 2005. Concerted action of poly(A) nucleases and decapping enzyme in mammalian mRNA turnover. Nat. Struct. Mol. Biol. 12: 1054-1063. 

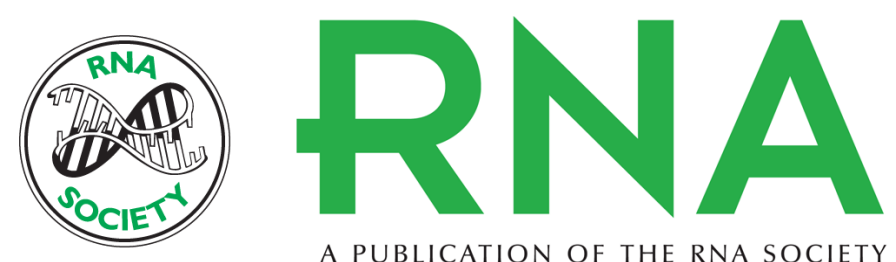

A PUBLICATION OF THE RNA SOCIETY

\section{Versatile applications of transcriptional pulsing to study mRNA turnover in mammalian cells}

Chyi-Ying A. Chen, Yukiko Yamashita, Tsung-Cheng Chang, et al.

RNA 2007 13: 1775-1786 originally published online August 29, 2007

Access the most recent version at doi:10.1261/rna.663507

References This article cites 44 articles, 23 of which can be accessed free at:

http://rnajournal.cshlp.org/content/13/10/1775.full.html\#ref-list-1

License

Email Alerting Receive free email alerts when new articles cite this article - sign up in the box at the Service top right corner of the article or click here. 